\title{
IMPREGNATED SEMICONDUCTOR SCINTILLATOR
}

\author{
SERGE LURYI \\ Electrical and Computer Engineering Department, University at Stony Brook \\ Stony Brook, NY 11794-2350, USA \\ Serge.Luryi@stonybrook.edu
}

\begin{abstract}
A semiconductor scintillation-type gamma radiation detector is discussed in which the gamma-ray absorbing semiconductor body is impregnated with multiple small direct-gap semiconductor inclusions of bandgap slightly narrower than that of the body. If the typical distance between them is smaller than the diffusion length of carriers in the body material, the photo-generated electrons and holes will recombine inside the impregnations and produce scintillating radiation to which the widegap body is essentially transparent. In this way it is possible to implement a semiconductor scintillator of linear dimensions exceeding $10 \mathrm{~cm}$.
\end{abstract}

Keywords: high-energy radiation detectors; semiconductor scintillator; direct-gap heterostructures.

\section{Introduction}

There are two large groups of solid-state radiation detectors, which dominate the area of ionizing radiation measurements, scintillation detectors and semiconductor diodes. The scintillators detect high-energy radiation through generation of light which is subsequently registered by a photo-detector that converts light into an electrical signal. Semiconductor diodes employ reverse biased $\mathrm{p}-\mathrm{n}$ junctions where the absorbed radiation creates electrons and holes, which are separated by the junction field thereby producing a direct electrical response. Both groups are extensively reviewed in the treatise by Knoll. ${ }^{1}$

In a recent paper, ${ }^{2}$ a new scintillation-type semiconductor detector was proposed in which high-energy radiation produces electron-hole pairs in a direct-gap semiconductor material that subsequently undergo interband recombination, producing infrared light to be registered by a photo-detector. Scintillators are not normally made of semiconductor material. The key issue in implementing a semiconductor scintillator is how to make the material essentially transparent to its own infrared light, so that photons generated deep inside the semiconductor slab could reach its surface without tangible attenuation.

An essential advantage of the semiconductor scintillator is that each scintillator slab can be supplied with its own, epitaxially grown photo-detector. Such slabs can then be stacked up without limit, thus not only increasing the active detector volume to accommodate large absorption length of high-energy radiation but also enabling the possibility of three-dimensional (3D) pixellation of Compton scatterings of the incident gamma photon. The 3D pixellation in turn enables rapid simultaneous determination of both the incident gamma-photon energy and the direction to its source. 
Two ways to accomplish transparency were discussed in Ref. 2. One way is based on heavy doping of bulk semiconductor with shallow impurities of one polarity type, preferably donors, so as to introduce the Moss-Burstein shift between the emission and the absorption spectra. ${ }^{\mathrm{a}}$

This approach is actively pursued in our laboratory at Stony Brook with InP used as the scintillator material. It turns out that heavy doping does improve the transparency of a semiconductor slab against interband absorption but it also increases the free-carrier absorption (FCA) in the doped material. ${ }^{3,4}$ Because of the high radiative efficiency of InP, minority carriers and photons exist in the $n$-type material as "interchangeable entities" (the effect is called the photon recycling). Every instance of interband absorption creates a new hole, which recombines (with a delay of 1 or $2 \mathrm{~ns}$ ) producing another photon in a random direction. Progression of the holes/photons to the epitaxial detector on the surface is a random walk. While the entity is a photon, its lifetime is limited by FCA and while it is a hole, the lifetime is limited by non-radiative processes, mainly Auger recombination. Both limitations are operative precisely because of the heavy doping, needed to effect the Burstein shift.

The resultant diffusion length of holes/photons in the individual InP scintillator slab at room temperature can be as high as $0.5 \mathrm{~mm}$ at the optimized doping level. This may be adequate for a system comprising thin $(<0.5 \mathrm{~mm})$ wafers, but it is clearly of considerable interest to implement a semiconductor scintillator with a thicker slab (say, an inch or larger), not relying on the Burstein shift for transparency.

Such a scintillator, in principle, can be obtained by following the other approach discussed in Ref. 2, in which the FCA is largely eliminated. The idea is to employ a heterostructure material, illustrated in Fig. 1. The epitaxially grown structure comprises two alternating materials that are lattice-matched to each other. The materials are assumed to have different energy gaps, $E_{\mathrm{G} 1}=E_{\mathrm{C} 1}-E_{\mathrm{V} 1}$ and $E_{\mathrm{G} 2}=E_{\mathrm{C} 2}-E_{\mathrm{V} 2}$, respectively, with the second material having the lower bandgap, $E_{\mathrm{G} 1}>E_{\mathrm{G} 2}$. The second material is assumed doped, while the first material is largely undoped. The distance between the narrow-gap wells is shorter than the diffusion length of carriers in the wide-gap material, which guarantees that all light emission occurs in the wells. In this case, the wider-gap material remains essentially transparent to the emitted photons. The essential idea is that the total volume occupied by the second material is small compared to that occupied by the first material. The ratio of these volumes defines a "duty cycle" factor $\delta$ and the absorption coefficient of the composite structure is reduced by this factor. For example, if a $2 \mu \mathrm{m}$-thick InP layers are alternated by a $20 \mathrm{~nm}$-thick layers of InGaAsP, the volume ratio is $100(\delta=0.01)$.

The heterostructure scintillator can be made several $\mathrm{cm}$ thick and is capable of efficient and fast operation even at room temperature. However, the fabrication of this device is not simple, to say the least. Indeed, most epitaxial growth techniques capable of nano-resolution are associated with a relatively slow growth process. The challenge is not

\footnotetext{
${ }^{a}$ In semiconductors with degenerate carrier concentrations, the edge of absorption is blue-shifted relative to the emission edge by the carrier Fermi energy. This effect underlies the operation of semiconductor lasers.
} 
only in the growth time that may span several days, but it is also very difficult to maintain the lattice matching of heterolayers in a thick structure. Even a small mismatch may lead to built-up stresses and a general deterioration of the material quality.

The InP based heterostructure is not the only system of interest in connection with the heterostructure scintillator as in Fig. 1. An attractive system to consider is CdTe with lattice-matched $\mathrm{Hg}_{\mathrm{x}} \mathrm{Cd}_{1-\mathrm{x}}$ Te wells. The bandgap of CdTe is $1.56 \mathrm{eV}$ and an admixture of mercury with $x \approx 0.07$ would provide a desired $100 \mathrm{meV}$ bandgap difference.

Fig. 1. Semiconductor scintillator by bandgap engineering (after Ref. 2). The heterostructure comprises two

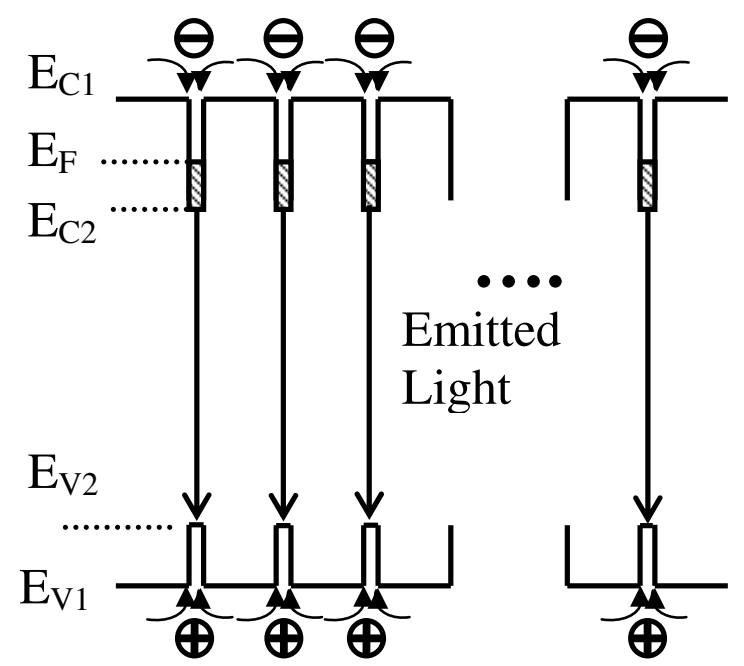

alternating materials, e.g., InP and InGaAsP quaternary alloy, lattice matched to InP, forming narrow wells, doped with donor impurities. Spacing between the wells, about $2 \mu \mathrm{m}$, is much larger than the well thickness but smaller than the diffusion length of carriers. Electrons and holes generated by the ionizing radiation rapidly diffuse to the wells and recombine there, producing scintillating radiation to which the composite material is substantially transparent. The main challenge is in the growth. It may be very difficult to maintain latticematched conditions between heterostructure components.

In the earlier paper, ${ }^{2}$ only lattice-matched implementations of the inhomogeneous scintillator were discussed. However, our patent application ${ }^{5}$ was more daring: the structure did not have to be layered or even planar: "the most general contemplated structure does not have to be layered. It may comprise droplets or impregnations of second material embedded in the matrix composed of the first material." The purpose of the present paper is to discuss this possibility in greater detail.

\section{Random Non-Planar Inclusions}

We now focus on the non-planar - and possibly far from the lattice-matched implementation of the inhomogeneous scintillator.

As before, we assume that the bandgap difference between the first and second materials is sufficiently large that light generated in the narrow-gap impregnations can be 
absorbed only by other impregnations, while the wide-gap matrix is essentially transparent. For an optically clean undoped semiconductor matrix $\left(1^{\text {st }}\right.$ material of bandgap $\left.E_{\mathrm{G} 1}\right)$ at room temperature, the interband absorption of light generated in the $2^{\text {nd }}$ material $\left(h v \approx E_{\mathrm{G} 2}\right)$ is quite negligible, provided $E_{\mathrm{G} 1}-E_{\mathrm{G} 2} \approx 100 \mathrm{meV}$.

Let us estimate the absorption by impregnations themselves. Let $\mu_{2} \approx 10^{4} \mathrm{~cm}^{-1}$ be the absorption coefficient of the scintillating light in the $2^{\text {nd }}$ material (possible additional suppression due to the Burstein effect is neglected here). Then the effective absorption coefficient is $\mu_{\text {eff }}=\mu_{2} \times \delta$, where $\delta$ is the duty cycle that controls the suppression of absorption. The essential point is that $\delta$ scales as the ratio of volumes, $\delta \approx(\mathrm{a} / d)^{3}$, where $a$ is the typical size of impregnations and $d$ the typical distance between them. Assuming that $a \approx 20 \mathrm{~nm}$ is large enough to avoid the quantum-dot regime ${ }^{6}$ (where the scintillation wavelength would strongly depend on the dot size) and $d \approx 2 \mu \mathrm{m}$ (limited by the requirement that the capture time of minority carriers is faster than their recombination in the lightly-doped matrix, as discussed in the next section), we have $\delta \approx 10^{-6}$, which means in practice that this type of absorption will not be relevant. Scattering and photon recycling will make the composite material look opaque - like glass with a fluctuating index - but not absorbing and hence not diminishing the scintillator performance. The residual absorption in the composite material will be in the wide-gap matrix, due to thermal free carriers, imperfections, such as unintentional doping, and so on.

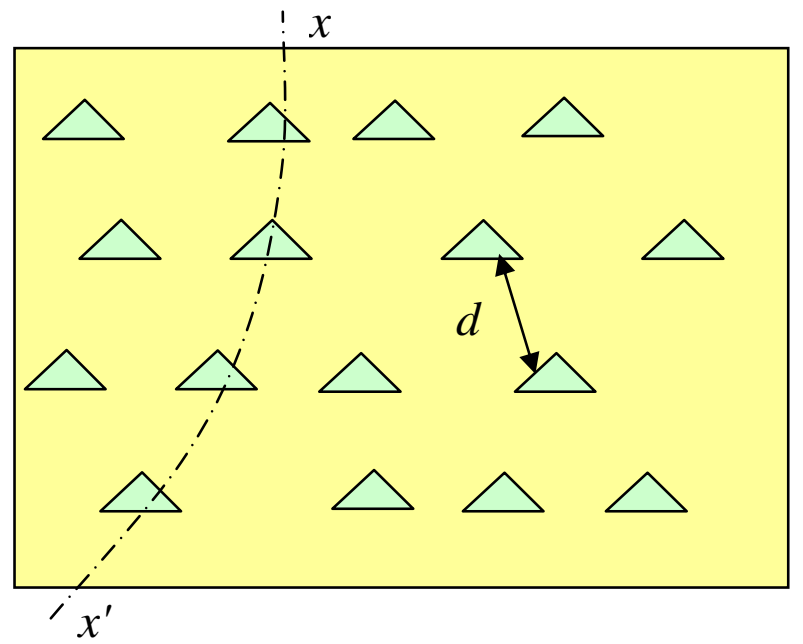

Fig.2. Impregnations or droplets of second material (shown as blue pyramids of size $a$ ) embedded in the matrix composed of the first material. The band structure along a curvilinear path $x x^{\prime}$ looks similar to that in Fig. 1, except for the arrangement of narrow-gap wells that is now irregular in general. The typical distance $d$ between the inclusions must be sufficiently small that the capture of minority carriers be faster than their recombination time in the lightly-doped matrix material. This ensures that recombination occurs mainly within the inclusions.

The doping polarity is primarily dictated by the convenience, but $p$-type doping appears preferable because of the higher mobility of minority carriers when these are electrons. Also the luminescence efficiency is often higher in $p$-type material. The corresponding band structure along the path $x x^{\prime}$ is shown in Fig. 3 . 
Realization of the fact that we do not need planarity of layers or lattice-matching relaxes our requirements to the crystal grower in the most essential way. One possibility for introducing inclusions is to employ the natural tendency of some material systems to phase-separate via spinodal decomposition during the crystal growth. For example, there is evidence for subband spectral emission in InGaN alloy films with small indium content, emission attributed to the tendency of indium to segregate during growth by vapor phase epitaxy. ${ }^{7}$ This type of segregation is unwelcome for most applications but in our context the effect may be beneficial, if it can be controlled.

At this time I believe that the preferred way to implement an impregnated semiconductor scintillator would involve a direct deposition of inclusions during interrupted epitaxial growth. How I envision this is discussed in Sect. 5. The technique appears to be not too dissimilar from the formation of self-organized quantum dots. ${ }^{8}$

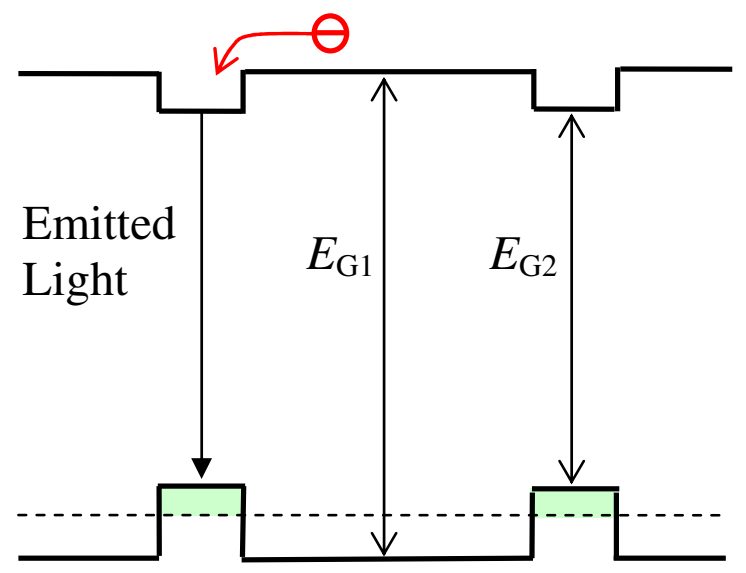

Fig. 3. Band diagram along the curvilinear path $x x^{\prime}$ of Fig. 2 for the case of p-doping, when electrons are the minority carriers.

\section{Efficient Capture of Minority Carriers}

First of all, we note that since we are no longer concerned with the Burstein shift-induced transparency, the dopant polarity can be either type, so long as it is the same polarity in both the matrix and the inclusions, cf. Fig. 3. A relevant consideration for the choice of doping is to enhance the luminescence efficiency that is often higher in $p$-type material. Another relevant consideration is the diffusion coefficient $D$ of the minority carriers, which favors minority electrons and therefore again $p$-type material. The diffusion coefficient comes into play when it is small, so that the capture of minority carriers by impregnations is diffusion limited, which corresponds to the situation when - due to efficient capture - the matrix material in the vicinity of impregnations is depleted of the minority carriers.

Below we shall refer to minority carriers as holes - left understood that they can be electrons as well, and in many cases preferably so. 
The efficient capture of holes is ensured if their lifetime $\tau_{1}$ in the lightly doped matrix

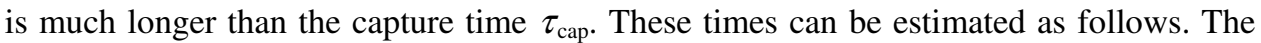
lifetime is limited by primarily by nonradiative processes because the radiative recombination of a hole will be followed by re-absorption of the interband photon. Owing to this effect of "photon recycling", we have $\tau_{1}>1 / B n \approx 1 \mu$ s, where we take the concentration $n \approx 10^{16} \mathrm{~cm}^{-3}$ and the radiative constant $B \approx 10^{-10} \mathrm{~cm}^{3} / \mathrm{s}$, typical for InP.

The capture time $\tau_{\text {cap }}$ can be written in the form ${ }^{9} \tau_{\text {cap }}=\tau_{0}+\tau_{\mathrm{D}}$, where

$$
\begin{aligned}
& \tau_{0}^{-1}=N \pi \tilde{a}^{2}\langle v\rangle \\
& \tau_{D}^{-1}=N 4 \pi D \tilde{a}
\end{aligned}
$$

Here $N \approx 1 / d^{3}$ is the density of inclusions, $D$ the diffusion coefficient of holes and $\langle v\rangle$ their thermal velocity. The latter two quantities are related by $D=\lambda\langle v\rangle / 3$ where $\lambda$ is the scattering mean-free path of holes. The effective capture amplitude $\tilde{a}$ corresponds to the radius of "no-return". It is assumed that once a hole is within a radius $\tilde{a}$ from the center of an impregnation, it is captured for sure. The capture amplitude $\tilde{a} \approx a+2 L_{\mathrm{D}}$ includes the Debye screening length, $L_{\mathrm{D}}{ }^{2}=\varepsilon k T / 4 \pi e^{2} n$, as illustrated in Fig. 4 .
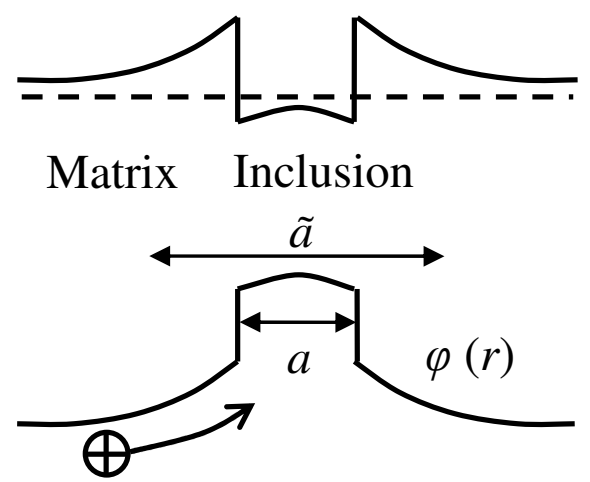

Fig. 4. Illustration of the effective minority-carrier capture amplitude $\tilde{a}$, associated with a single inclusion. It is assumed that both the lightly-doped matrix and the inclusions have the same polarity of doping. It is shown here as $n$-type, but $p$-type in some cases may be preferable.

The relative importance of the two terms in Eq. (1) depends on the ratio $\lambda / \tilde{a}$. The first term (1a) dominates when $\lambda>>/ \tilde{a}$. The opposite limit corresponds to diffusionlimited capture. ${ }^{9}$ For $n \approx 10^{16} \mathrm{~cm}^{-3}$ one has $L_{\mathrm{D}} \approx 40 \mathrm{~nm}$ and hence $\tilde{a} \approx 100 \mathrm{~nm}$. The scattering mean-free path for minority carriers is of the same order, $\lambda \approx 100 \mathrm{~nm}$, which means that both terms in (1) are comparable. For $d \approx 1 \mu \mathrm{m}$ at room temperature the estimated capture time is at most several nanoseconds, i.e., $\tau_{\text {cap }}<<\tau_{1} \approx 1 \mu$ s. Thus, the requirement of efficient capture of holes does not impose stringent limitations. We can afford to space out the impregnations be several microns. To be sure, if the matrix material has unintentional doping on the level of $n \approx 10^{17} \mathrm{~cm}^{-3}$, then the lower limit for $\tau_{1}$ 
shrinks to $0.1 \mu$ s and at the same time we will not get as much benefit from the screening length $L_{\mathrm{D}} \approx 12 \mathrm{~nm}$. Nevertheless, we should still be able to space out the inclusions by as much as $d \approx 1 \mu \mathrm{m}$ without introducing a tangible carrier loss.

The diffusion-limited capture of minority carriers is an old standing problem, first considered by Langevin and subsequently by Pekar and others. References to these classical papers can be found in the beautiful monograph ${ }^{9}$ by Abakumov, Perel and Yassievich. In recent years the subject has been extensively discussed in connection with quantum-dot infrared photodetectors. ${ }^{10,11}$ In particular, the QDIP literature addresses the effect of a potential barrier at the interface between the two materials. As far as I am aware, the issue of minority carrier trapping at the interface has not been treated theoretically. The possibility of interface trapping should certainly be of concern but it can probably be resolved only by experimentation.

\section{Possible Material Systems}

We need a compatible, but not necessarily lattice-matched binary system, where at least the $2^{\text {nd }}$ material is a direct-gap semiconductor. Furthermore, an important requirement is the possibility of implementing a photoreceiver system, epitaxial on the host material. Consider several examples:

(1) InP matrix, bandgap $E_{\mathrm{G} 1}=1.344 \mathrm{eV}$, lattice constant $a_{1}=0.587 \mathrm{~nm}$. The ternary alloy $\operatorname{InAs}_{\mathrm{y}} \mathrm{P}_{1-\mathrm{y}}$ will have $E_{\mathrm{G} 2}(y) \approx E_{\mathrm{G} 1}-0.99 y($ in $\mathrm{eV})$. At the desired $100 \mathrm{meV}$ difference (achieved for $y \approx 0.1$ ) the lattice constant $a_{2}[\mathrm{~nm}]=a_{1}+0.019 y$ will be about $0.3 \%$ mismatched. The epitaxial photoreceiver feasibility has been proven in our laboratory by successful implementation of quaternary InGaAsP pin diodes lattice-matched to $\mathrm{InP}$ (to be reported).

(2) CdTe matrix, $E_{\mathrm{G} 1}=1.56 \mathrm{eV}$, lattice constant $a_{1}=0.648 \mathrm{~nm}$. The bandgap of the ternary alloy $\mathrm{Hg}_{\mathrm{x}} \mathrm{Cd}_{1-\mathrm{x}} \mathrm{Te}$ is $E_{\mathrm{G} 2}(x) \approx E_{\mathrm{G} 1}-1.57 x$ (in $\mathrm{eV}$ ), so that desired $100 \mathrm{meV}$ bandgap difference is achieved at $x \approx 0.07$. Since the ternary alloy is lattice-matched at all $x$, it should be feasible to implement epitaxial pin diodes on the surface of $\mathrm{CdTe}$, but the quality of such diodes remains to be proven. In this regard, very encouraging are recent reports ${ }^{13}$ of infrared avalanche photodiodes implemented in $\mathrm{HgCdTe}$.

(3) GaAs with dilute-nitride inclusions. Small admixtures (less than atomic percent) of nitrogen are known to narrow the bandgap of GaAs while at the same time somewhat shrinking the lattice constant. ${ }^{12}$ This appears to be a very compatible system and the lattice mismatch may be actually an advantage for the formation of inclusions. One should be clear, however, that it is difficult to implement a lattice-matched photodiode epitaxial on GaAs that would be sensitive to wavelengths longer than $\mathrm{GaAs}$ fundamental emission $(860 \mathrm{~nm})$. One possibility of interest in this regard is to consider the dilute-nitride InGaAs-N system. An InGaAs alloy also has a narrower bandgap - but with a larger lattice constant than GaAs. Therefore, in principle, the quaternary dilute-nitride InGaAs-N alloy can remain lattice matched to GaAs, while 
satisfying the requirements for epitaxial detector of light generated in the ternary GaAs-N inclusions.

(4) GaAs matrix can also be considered with InP inclusions. From the standpoint of bandgap difference this is an ideal system with GaAs gap of $1.44 \mathrm{eV}$ and InP inclusions at $1.35 \mathrm{eV}$. The lattice mismatch in our case is a blessing as it should force the Stranski-Krastanov growth of InP inclusions. However, one would again have to rely on the quaternary dilute-nitride InGaAs-N for implementation of an epitaxial detector of light generated in InP inclusions.

(5) The most widely used system that produces "self-organized" (quantum) dots is GaAs or AlGaAs with InGaAs inclusions, used in the quantum-dot laser technology. However, the $\mathrm{In}_{\mathrm{x}} \mathrm{Ga}_{1-\mathrm{x}}$ As alloy grown on InP goes into the 3D (Stranski-Krastanov) growth mode typically when $x>0.3$.

From the standpoint of Compton interaction, GaAs has about the same absorption efficiency as InP (both have 64 electrons per unit cell, which makes the mean-free path of $\gamma$ photons with respect to Compton scattering approximately equal to that of $\mathrm{Ge}$, which also has 64 electrons per unit cell). In contrast, CdTe with 100 electrons per unit cell is much more effective. This is very important and one should deploy much effort to ascertain the feasibility of $\mathrm{CdTe} / \mathrm{HgCdTe}$ composite scintillator system. However, this system may have other challenges. For example, its radiative efficiency may not be as high as that of InP. Also, it may be more difficult to implement a low-noise epitaxial surface photodiode array on CdTe, compared to that on the surface of InP.

\section{What Do We Need From the Crystal Grower?}

First and foremost, we need the ability to grow thick structures, on the millimeter scale. Figure 5 illustrates the possible approach to the growth.

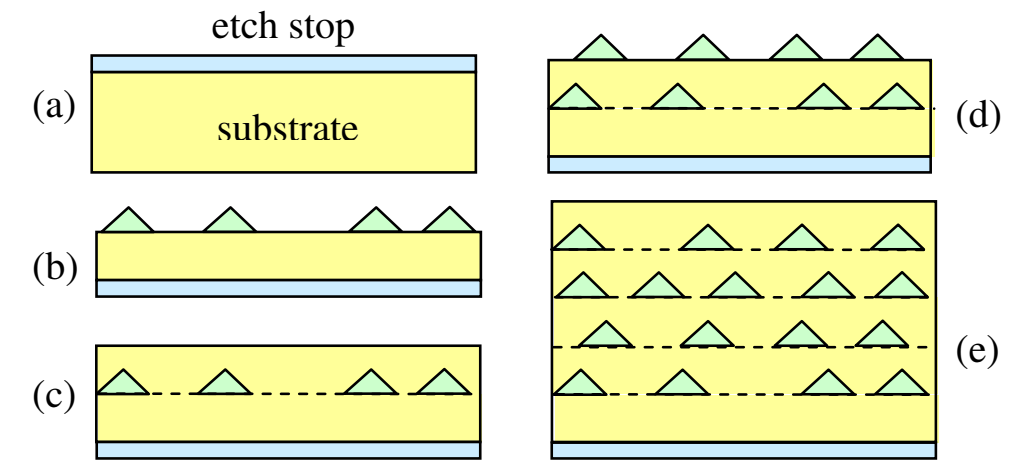

Fig. 5. Illustration of the material fabrication sequence. (a) Growth begins on a substrate (yellow) covered with an etch stop layer (blue) that will enable ultimate removal of the substrate. For example, $0.1 \mu \mathrm{m}$ InGaAs layer can be used as an etch-stop to remove an entire InP substrate; $(b)$ Initial epitaxial growth 2-3 $\mu \mathrm{m}$ of first (widegap) material and deposition of microcrystalline grains of second material with partial coverage. Substrate is no longer shown but it is still there! (c) Overgrowth with another 2-3 $\mu \mathrm{m}$ of first material; $(d)$ Second deposition of microcrystalline grains of second material with partial coverage; (e) After multiple repetition of steps (c) and (d) a thick enough structure is grown (like in Fig. 2) and the substrate is removed using the etch-stop layer. 
The structures should be grown in steps of 2 to 3 microns, followed by the deposition of possibly lattice-mismatched non-covering grains of lower-bandgap luminescent material. Growth of the matrix material should be fast, of order 1 micron per minute, so that the entire growth can be accomplished in less than one day. It seems that hydrate vapor phase epitaxy $^{14}$ (HVPE) is the preferred growth method but liquid phase epitaxy ${ }^{15}$ (LPE) may also be a plausible technique.

The contemplated fabrication sequence is illustrated in Fig. 5. The growth of a freestanding structure is contemplated on a substrate that can be removed after growth. For this purpose, one can introduce an etch-stop layer of high selectivity, shown in Fig. 5a. Homoepitaxial layers are deposited by a rapid growth technique several microns at a time. The lattice-mismatched impregnations are then introduced in such a way that they form isolated islands to be subsequently overgrown by the matrix layers. The matrix remains therefore homoepitaxial at all times during the growth, seeded by the free surface between impregnations.

\section{Conclusion}

Needless to say, the implementation of an impregnated scintillator structure remains a long shot proposition. Nevertheless, it offers a very high pay-off, especially in homeland security applications, where the development of an efficient semiconductor scintillator offers excellent opportunities for isotope discrimination and directional resolution. Sequential growth incorporating disconnected inclusions relieves some of the problems associated with the layered heterostructure growth, because in our case the matrix remains homoepitaxial and stresses are not expected to develop in thick film growth. The crucial issue is the speed of epitaxial growth that should exceed $1 \mu \mathrm{m} / \mathrm{min}$ to enable practical fabrication of millimeter-thick structures. In my opinion, the search for the right material combination and sound fabrication technology merits a concerted effort in several directions, some of which have been delineated in this purely conceptual paper.

Once a particular material system is chosen, there will be obviously many questions to resolve, both experimentally and theoretically. An immediate question to address is whether the lattice-mismatch driven decomposition is more efficient in our context than spinodal decomposition. Will there be significant correlations between inclusions in a single layer and what effect such correlations will have on the scintillator performance? Another interesting and possibly important effect is the competition between photon collection and minority carrier collection at the surface photo-diode structures.

\section{Acknowledgments}

I am grateful to A. Kastalsky, N. Lifshitz and A. Subashiev for many useful discussions. This work has been partially supported by the Domestic Nuclear Detection Office (DNDO) of the Department of Homeland Security and partially by the New York State Office of Science, Technology and Academic Research (NYSTAR) through the Center for Advanced Sensor Technology (Sensor CAT) at Stony Brook. 


\section{References}

1. Glenn F. Knoll, Radiation Detection and Measurement, $3^{\text {rd }}$ ed. (Wiley, New York, 2000).

2. A. Kastalsky, S. Luryi and B. Spivak, Semiconductor high-energy radiation scintillation detector, Nucl. Instr. Meth. Phys. Res. A 565, 650-656 (2006).

3. W. P. Dumke, M. R. Lorenz and G. D. Pettit, Intra- and Interband Free-carrier Absorption and the Fundamental Absorption Edge in $n$-Type InP, Phys. Rev. B1, $4668-4673$ (1970).

4. F. K. Reinhart, Direct determination of the free-carrier injection density, the free-carrier absorption, and the recombination factors in double heterostructure diodes by optical phase measurements, J. Appl. Phys. 97, 123536:1-13 (2005).

5. A. Kastalsky, S. Luryi and B. Spivak, US Patent 7,265,354 (issued Sept 2007).

6. A. D. Yoffe, Semiconductor quantum dots and related systems: electronic, optical and related properties of low dimensional systems, Adv. Phys. 50, 1-208 (2001).

7. M. S. Shur, private communication.

8. Todd Steiner (editor), Semiconductor Nanostructures for Optoelectronic Applications (Artech House, Boston 2004).

9. V.Abakumov, V.Perel and I.Yassievich, Nonradiative Recombination in Semiconductors (Elsevier Science, Amsterdam, 1991).

10. H.Lim, B.Movaghar, S.Tsao, M.Taguchi, W.Zhang, A.A.Quivy, and M.Razeghi, Gain and recombination dynamics of quantum-dot infrared photodetectors, Phys. Rev. B74, 205321:1-8 (2006).

11. N.Vagidov, A.Sergeev and V.Mitin, Infrared quantum-dot detectors with diffusion-limited capture, Int. J. High Speed Electronics and Systems 17, 585-591 (2007); see also L.H.Chien, A.Sergeev, N.Vagidov, and V.Mitin, Hot electron transport in quantum-dot photodetectors (present volume).

12. Mohamed Henini, Dilute Nitrides Semiconductors (Elsevier, Oxford, U.K., 2005).

13. J. Beck, C. Wan, M. Kinch, J. Robinson, P. Mitra, R. Scritchfield, F. Ma, and J. Campbell, The HgCdTe electron avalanche photodiode, J. Electron. Mat. 35, 1166-1173 (2006).

14. S. Lourdudoss and O. Kjebon, Hydride Vapor Phase Epitaxy Revisited, IEEE J. Selected Topics Quant. Electronics 3, 749-767 (1997).

15. Peter Capper and Michael Mauk (editors), Liquid Phase Epitaxy of Electronic, Optical and Optoelectronic Materials (Wiley, Chichester, U.K., 2007). 